\title{
A retrospective study detects a novel variant of porcine epidemic diarrhea virus in England in archived material from the year 2000
}

Falko Steinbach ${ }^{\text {Corresp.. }}{ }^{1}$, Akbar Dastjerdi ${ }^{1}$, Julie Peake ${ }^{1}$, S Anna La Rocca ${ }^{1}$, Frank P Tobin ${ }^{2}$, Jean-Pierre Frossard ${ }^{1}$, Susanna Williamson ${ }^{3}$

1 Virology, Animal and Plant Health Agency, Addlestone, Surrey, United Kingdom

2 Holmefield Farm Services Ltd, Murton, Yorkshire, United Kingdom

3 Surveillance Intelligence Unit, Animal and Plant Health Agency, Bury St Edmunds, Suffolk, United Kingdom

Corresponding Author: Falko Steinbach

Email address: falko.steinbach@apha.gsi.gov.uk

Outbreaks of porcine epidemic diarrhea (PED) were first recorded in England in the 1970s and continued to be confirmed until 2002. Retrospective analysis of archived material from one of the last confirmed cases in England in the year 2000 demonstrates the previous existence of a very diverse PED virus strain. Following the outbreaks of PED in North America in 2013, there has been renewed interest in phylogenetic analysis of sequences from PEDV strains worldwide. There is a gap in the available sequence data between the mid 1980s and the mid 2000s. This work is an example of how this gap can be at least partially filled by the examination of archived material. 
1 A retrospective study detects a novel variant of porcine epidemic diarrhea virus in England in 2 archived material from the year 2000

3

4

5 Falko Steinbach ${ }^{\mathrm{a}}$, Akbar Dastjerdi ${ }^{\mathrm{a}}$, Julie Peake ${ }^{\mathrm{a}}$, S. Anna La Rocca ${ }^{\mathrm{a}}$, Frank Tobin ${ }^{\mathrm{b}}$, Jean-Pierre

6 Frossard $^{\mathrm{a}}$, Susanna Williamson ${ }^{\mathrm{c}}$

7

8 Animal and Plant Health Agency, Weybridge, Addlestone, UK ${ }^{\mathrm{a}}$; Holmefield Farm Services Ltd

9 Murton Lane, Murton, York, UK ${ }^{\mathrm{b}}$; Animal and Plant Health Agency, Bury St Edmunds, UK

10

11

12 Correspondending author: Falko Steinbach, falko.steinbach@apha.gsi.gov.uk.

13 


\section{Abstract}

15 Outbreaks of porcine epidemic diarrhea (PED) were first recorded in England in the 1970s and continued to be confirmed until 2002. Retrospective analysis of archived material from one of

17 the last confirmed cases in England in the year 2000 demonstrates the previous existence of a very diverse PED virus strain. Following the outbreaks of PED in North America in 2013, there has been renewed interest in phylogenetic analysis of sequences from PEDV strains worldwide. There is a gap in the available sequence data between the mid 1980s and the mid 2000s. This work is an example of how this gap can be at least partially filled by the examination of archived

22 material.

\section{Introduction}

25 Porcine epidemic diarrhea virus (PEDV), an Alphacoronavirus in the order Nidovirales, was first

26 described in England in the 1970s and subsequently emerged across most of Europe (Wood,

27 1977; Pensaert \& de Bouck, 1978). While the virus reached southeast Asia in the 1980s, reports

28 of PED declined in Europe and no cases have been reported from the field in England since 2002

29 (Williamson et al., 2013). In recent years however, variant strains of PEDV have emerged in

30 China causing high mortality in sucking piglets and spreading rapidly between farms (Sun et al.,

31 2012). In 2013 these virulent PEDV strains were reported in pig herds in the United States

32 (Stevenson et al., 2013). This was followed by a report from Ohio of another PEDV strain

33 characterised by insertions/deletions in the S-gene, known as the INDEL strain, OH851 being the

34 prototype (Wang, Byrum \& Zhang, 2014), associated with reportedly milder disease in the field.

35 Most recently INDEL PEDV strains have been detected in outbreaks of diarrhoea in Western

36 Europe, including Germany, the Netherlands, France and Italy (Hanke et al., 2015; EFSA, 2016). 
37 In parallel the PEDV strain associated with higher virulence in Asia and North America was

38 detected in the Ukraine (Dastjerdi et al., 2015).

39 The molecular characterization of PEDV has been established (Huang et al., 2013). The virus is

40 an enveloped, positive-sense, single-stranded RNA coronavirus possessing a genome of $\approx 28$

41 kilobases. To classify strains involved in the previous and recent outbreaks, a phylogenetic

42 nomenclature using genogroups or clades has been suggested (Huang et al., 2013). Markers for

43 pathogenicity are poorly defined for coronaviruses, thus any focused sequencing of the S1 region

44 only, for example, might introduce unreasonable bias into the analysis.

45 This paper reports the results of analysis of the PEDV from a historic field case in England,

46 including comparisons with currently circulating North American/Asian viruses, INDEL strains

47 and the European prototype strain CV777 (Kocherhans et al., 2001).

Materials and methods

\section{Clinical case history and diagnostic investigation}

51 Between 1999 and 2002, the national veterinary diagnostic database for GB (VIDA) recorded 13

52 diagnoses of PED on nine pig units in five counties of England, with the last diagnosis being

53 made in 2002 and none since. Unfortunately the archived data does not allow us to draw

54 epidemiological links between these cases. Archived material was only available from one

55 outbreak from October 2000; a finishing unit in Yorkshire reporting an outbreak of diarrhoea.

56 Three fecal samples were submitted at that time, and a diagnosis of PED was made by testing the

57 samples using a duplex RT-PCR (primer sequences TTTATTCTGTCACGCCATGT and

58 CCAGATTTACAAACACCTATGTTA spanning a 199bp fragment) designed to detect and

59 discriminate the S gene from both PEDV and transmissible gastroenteritis virus (TGEV). All 
60 three samples tested positive for PEDV. Differential diagnostic testing for Salmonella and

61 Brachyspira species by selective culture identified Salmonella enterica ser. Typhimurium phage

62 type 193 only after enrichment culture in one of the three fecal samples, negating a diagnosis of

63 salmonellosis or swine dysentery. One of the samples, from a 14-week-old pig, was retained in

64 the cryostore archive, and was retrieved for further analysis in 2014.

65 Analysis of the archived PEDV-positive faeces

66 The original positive PEDV PCR result was re-confirmed on the archived faeces, and also using

67 a further in-house PCR targeting the $\mathrm{N}$ gene. The initial PCR amplicon was subjected to

68 conventional Sanger sequencing to confirm the detection of PEDV. Upon recognition of the

69 significant differences to other PEDV strains, next generation full genome sequencing was

70 carried out to avoid the necessity of designing novel primers in multiple rounds of Sanger

71 sequencing for such a large RNA genome. In brief, the extracted PEDV RNA was subjected to

72 DNase digestion and used as template for cDNA generation using a cDNA Synthesis System

73 (Roche) for preparation of sequencing libraries using a Nextera XT kit (Illumina, San Diego,

74 USA). Paired end sequencing was performed on an Illumina MiSeq. Finally, the consensus

75 sequence was obtained by de novo assembly using Velvet 1.2.10 as previously described (Miller

76 et al., 2010) and re-evaluated using the templated genome assembly function of the SeqMan

77 NGen v13 software (DNASTAR Inc. Madison, USA). The consensus sequence was generated

78 from 321425 sequence reads; considering length of each read the average coverage for each base

79 pair is 1341.15 , which is well above the optimum coverage. For phylogenetic analysis,

80 comparisons were made with published spike protein amino acid sequences of 32 other PEDV

81 strains and two other coronaviruses, and similarly for the ORF1a/b nucleotide sequence.

82 Sequence alignments were performed using the Clustal W algorithm, and phylogeny was 
83 performed using the maximum likelihood method with 1000 bootstrap replications, both using

84 MEGA version 6 (Tamura et al., 2013).

85

\section{Results}

87 PCR testing of the faecal sample from 2000 confirmed the presence of PEDV nucleic acid and

88 the absence of TGEV, indicating involvement of PEDV in the outbreak of diarrhoea in the

89 finishing pigs.

90 As shown in the figure, phylogenetic analysis of the consensus whole genome sequence obtained

91 directly from the archived faecal sample indicates that the virus, while clearly belonging to the

92 PEDV species, lies distinct from any of the genogroups described by Huang et al. in 2013.

93 At the amino acid level, as shown in the table, proteins from this England-1-2000 strain vary in

94 similarity from $91.5 \%$ to $96.3 \%$ compared to those of the CV777 strain. At the nucleotide level,

95 similarities for the ORF1a/b region vary from $95.1 \%$ to $96.3 \%$ when compared to the other

96 PEDV sequences analysed. While this strain is significantly different to other PEDV strains

97 known so far, it is in no part more closely related to other known coronaviruses, thus does not

98 resemble the result of inter-species recombination such as described for the Italian swine enteric

99 coronavirus (Boniotti et al., 2016). The complete genome sequence of PEDV England-1-2000

100 has been deposited at GenBank under the accession number KU836638.

101

\section{Discussion}

103 While PEDV England-1-2000 was significantly different from the European prototype CV777

104 from the early 1980s (Ducatelle et al., 1981), current and established PCR tests in 2000 were

105 able to detect the virus without modification, suggesting that similar strains were not simply 
106 missed due to lack of diagnostic potential. The virus also differs from the virulent North

107 American/Asian and the INDEL variants such as OH851 currently circulating in Western

108 Europe. The lack of other published PEDV sequences from historic outbreaks of PED to the

109 2000s prevents further comparisons, but this clearly represents a genotype not described

110 previously.

111 No detailed morbidity or mortality data are available from records of the PED outbreak from

112 which this virus was identified, but a previous diagnosis of PED was made on the farm in an

113 earlier batch of finishing pigs in November 1999. The isolation of Salmonella Typhimurium in

114 just one of the three fecal samples by enrichment culture only, and lack of isolation of

115 Brachyspira species from the samples, indicates no significant involvement of salmonellosis or

116 Brachyspira-related colitis in this diarrhea outbreak. In general, PED outbreaks at that time

117 caused watery diarrhea without mortality in growing pigs and sows rather than piglets, and

118 tended to spread rapidly through the herd. This clinical presentation may, in part, reflect

119 immunity through natural infection in the national herd at that time. Unfortunately, no viable

120 virus could be recovered from the limited amount of material available after the prolonged

121 storage period to assess the relative virulence of this strain in experimental infections.

122 This finding shows the presence of a diverse field PEDV strain in England at the turn of the

123 century, the pathogenicity of which requires further investigation that will only be possible upon

124 rescuing the strain via reverse genetics. Outbreaks of PED were diagnosed in GB until 2002

125 although the annual incidence of laboratory confirmed outbreaks was quite low, and there is no

126 evidence that this strain is still present as a cause of disease in GB (EFSA, 2016). This report

127 highlights the merit of further investigation of archived material from Europe and elsewhere to 
128 establish the degree of heterogeneity of historic PEDV strains and determine whether this

129 England-1-2000 strain was typical of those circulating at the time in Europe.

130

131 Acknowledgements:

132 We are grateful to Sonia Zuñiga and Luis Enjuanes, Madrid for assisting attempts to isolate this

133 strain, and to Richard J. Ellis for NGS sequencing.

134 


\section{References}

136 Boniotti MB, Papetti A, Lavazza A, Alborali G, Sozzi E, Chiapponi C, Faccini S, Bonilauri

137 P, Cordioli P, Marthaler D. 2016. Porcine epidemic diarrhea virus and discovery of a

138 recombinant swine enteric coronavirus, Italy. Emerg Infect Dis. 22:83-87.

139 Dastjerdi A, Carr J, Ellis RJ, Steinbach F, Williamson S. 2015. Porcine epidemic diarrhea

140 Virus among farmed pigs, Ukraine. Emerg Infect Dis. 21:2235-2237.

141 Ducatelle R, Coussement W, Pensaert MB, Debouck P, Hoorens J. 1981. In vivo

142 morphogenesis of a new porcine enteric coronavirus, CV 777. Arch Virol. 68:35-44.

143 European Food Safety Authority. 2016. Collection and review of updated scientific

144 epidemiological data on porcine epidemic diarrhea. EFSA Journal. 14(2):4375.

145 Hanke D, Jenckel M, Petrov A, Ritzmann M, Stadler J, Akimkin V, Blome S, Pohlmann A,

146 Schirrmeier H, Beer M, Höper D. 2015. Comparison of porcine epidemic diarrhea viruses

147 from Germany and the United States, 2014. Emerg Infect Dis. 21(3):493-496.

148 Huang YW, Dickerman AW, Piñeyro P, Li L, Fang L, Kiehne R, Opriessnig T, Meng XJ.

149 2013. Origin, evolution, and genotyping of emergent porcine epidemic diarrhea virus strains in

150 the United States. mBio. 4(5):e00737-13. doi: 10.1128/mBio.00737-13.

151 Kocherhans R, Bridgen A, Ackermann M, Tobler KG. 2001. Completion of the porcine

152 epidemic diarrhea coronavirus (PEDV) genome sequence. Virus Genes. 23(2):137-144.

153 Miller JR, Koren S, Sutton G. 2010. Assembly algorithms for next-generation sequencing data.

154 Genomics. 95(6):315-327.

155 Pensaert MB, de Bouck P. 1978. A new coronavirus-like particle associated with diarrhea in 156 swine. Arch Virol. 58(3):243-247. 
157 Stevenson GW, Hoang H, Schwartz KJ, Burrough ER, Sun D, Madson D, Cooper VL,

158 Pillatzki A, Gauger P, Schmitt BJ, Koster LG, Killian ML, Yoon KJ. 2013. Emergence of

159 Porcine epidemic diarrhea virus in the United States: clinical signs, lesions, and viral genomic

160 sequences. J Vet Diagn Invest. 25(5):649-654.

161 Sun RQ, Cai RJ, Chen YQ, Liang P, Chen D, Song C. 2012. Outbreak of porcine epidemic

162 diarrhea in suckling piglets, China. Emerg Infect Dis. 18(1):161-163.

163 Tamura K, Stecher G, Peterson D, Filipski A, Kumar S. 2013. MEGA6: Molecular

164 Evolutionary Genetics Analysis version 6.0. Mol Biol Evol. 30:2725-2729.

165 Wang L, Byrum B, Zhang Y. 2014. New variant of porcine epidemic diarrhea virus, United

166 States. Emerg Infect Dis. 20(5):917-919.

167 Williamson S, Strugnell B, Thomson J, Webster G, McOrist S, Clarke H, Armstrong D.

168 2013. Emergence of porcine epidemic diarrhoea in pigs in the USA. Vet Record. 173:146-148.

169 Wood EN. 1977. An apparently new syndrome of porcine epidemic diarrhea. Vet Rec. 100:243170244.

171 


\section{Table $\mathbf{1}$ (on next page)}

Amino acid identity of PEDV England-1-2000 compared to reference strains of European and US PEDV 
1

\begin{tabular}{|c|c|c|c|c|c|c|}
\hline Protein: & ORF1a/b & $S$ & ORF3 & $E$ & $\mathrm{M}$ & $\mathrm{N}$ \\
\hline \multicolumn{7}{|l|}{$\%$ identity to } \\
\hline $\begin{array}{l}\text { CV777 } \\
\text { (AF353511): }\end{array}$ & $96.3 \%$ & $92.0 \%$ & $91.5 \%$ & $93.4 \%$ & $94.2 \%$ & $94.8 \%$ \\
\hline \multicolumn{7}{|l|}{$\%$ identity to } \\
\hline $\begin{array}{l}\text { Colorado } 2013 \\
\text { (KF272920): }\end{array}$ & $96.2 \%$ & $89.7 \%$ & $91.1 \%$ & $92.1 \%$ & $93.4 \%$ & $93.4 \%$ \\
\hline \multicolumn{7}{|l|}{$\%$ identity to } \\
\hline $\begin{array}{l}\text { OH851 } \\
\text { (KJ399978): }\end{array}$ & $96.2 \%$ & $91.7 \%$ & $91.5 \%$ & $92.1 \%$ & $93.4 \%$ & $93.2 \%$ \\
\hline
\end{tabular}

2 


\section{Figure 1 (on next page)}

\section{Molecular phylogeneticanalysis of PEDV sequences}

A) Molecular phylogenetic analysis of 33 PEDV spike protein amino acid sequences. The evolutionary history was inferred by using the Maximum Likelihood method. The tree with the highest log likelihood (-

10946.4157) is shown. Initial trees for the heuristic search were obtained by applying the Neighbor-Joining method to a matrix of pairwise distances estimated using a JT model. A discrete Gamma distribution was used to model evolutionary rate differences among sites ( 5 categories $(+G$, parameter $=1.4131)$ ). The rate variation model allowed for some sites to be evolutionarily invariable $([+1], 0 \%$ sites).

B) Molecular phylogenetic analysis of 33 PEDV ORF1 nucleotide sequences. The evolutionary history was inferred by using the Maximum Likelihood method based on the Tamura-Nei model. The tree with the highest log likelihood (-85920.7638) is shown. Initial tree(s) for the heuristic search were obtained by applying the Neighbor-Joining method to a matrix of pairwise distances estimated using the Maximum Composite Likelihood (MCL) approach.

The tree is drawn to scale, with branch lengths measured in the number of substitutions per site. The trees are drawn to scale, with branch lengths measured in the number of substitutions per site. The percentage of trees in which the associated taxa clustered together is shown next to the branches. Evolutionary analyses were conducted in MEGA6 (Tamura et al., 2013). The GenBank accession numbers, country and year of isolation are indicated, along with the genogroups as described by Huang et al. (2013). Porcine respiratory coronavirus (PRCV) and transmissible gastroenteritis (TGEV) virus sequences are shown for reference. The prototype PEDV strains CV777, OH851, Colorado 2013 and England-1-2000 strains are underlined. 


\section{PeerJ}

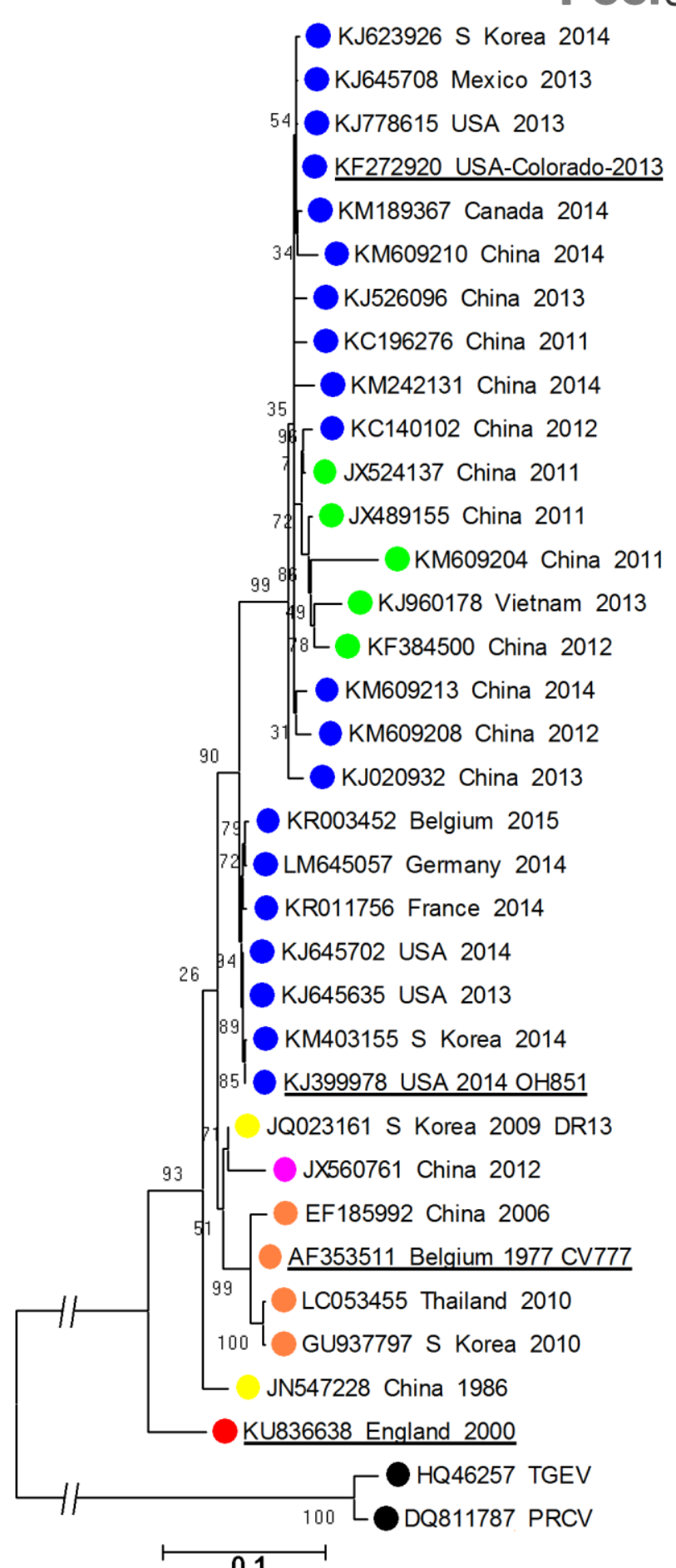

$2 a$

$2 b$

$2 a$

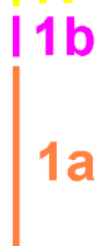

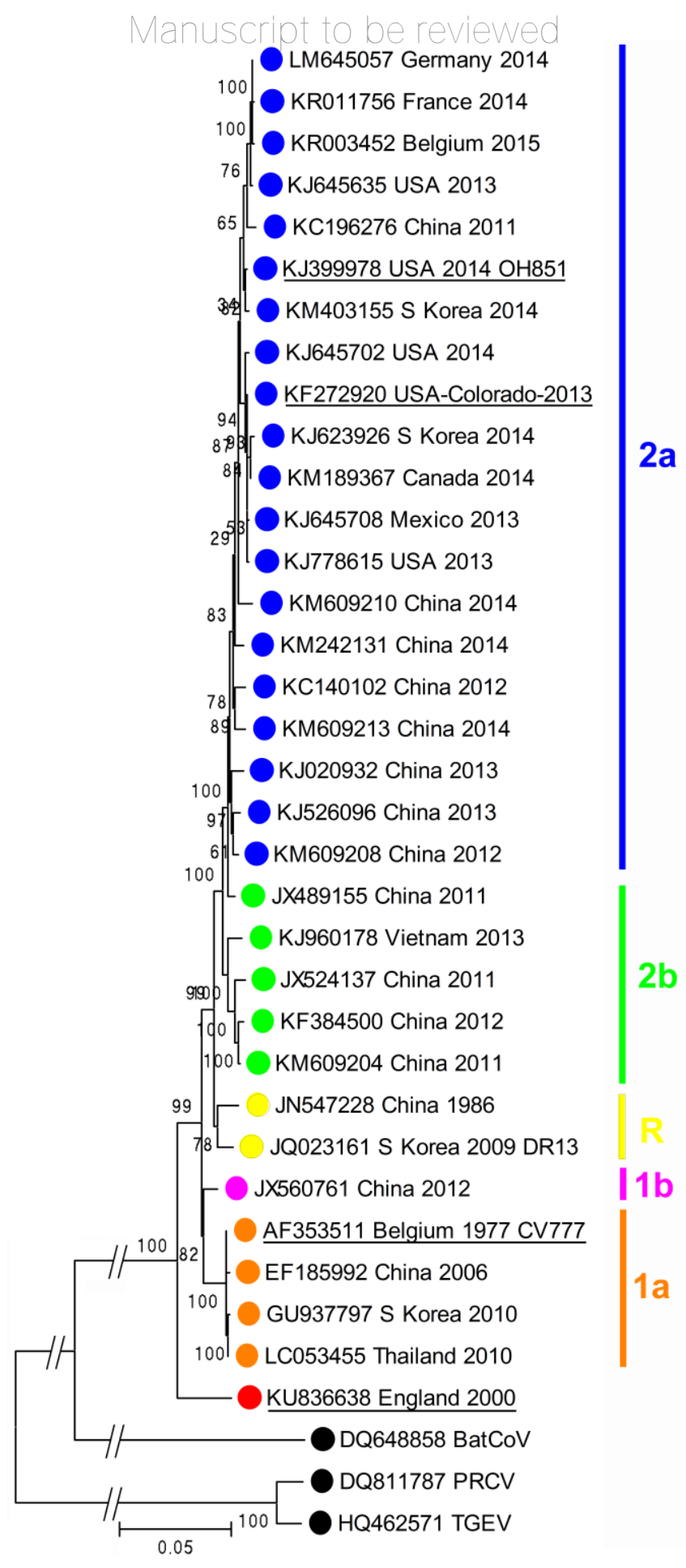

